\title{
1 Ethical issues concerning informed consent in translational/clinical research and vaccination
}

\section{Alberto García and Mirko Garasic}

Improving the health literacy of patients in relation to medical practices and research is essential for upholding the principle of respect for autonomythat is, respecting the patient's ability to make self-governed choices regarding medical interventions or research participation that reflects the patient's beliefs and values.

This report ${ }^{1}$ provides a full review of informed consent challenges (i.e. ethical gaps, barriers and priority needs) that are unique to certain vulnerable groups, namely, preadolescents, adolescents, and pregnant women, with a specific emphasis on how neurobioethical, multicultural and interreligious variables should be taken into account when assessing the appropriateness of the current documents relying on the notion of informed consent. In exploring how we are to improve the process of obtaining informed consent, we will also highlight the relevance of bias and privacy in the debate. The objective is to offer recommendations on how these gaps, barriers and challenges may be solved or avoided in the future.

There are two categories of challenges. The first category comprises challenges that are patient-centred, which prevent a research subject from fully comprehending the disclosed information. The second category comprises challenges that are process-centred, which are procedural barriers that prevent obtaining truly informed consent from prospective patients.

The types of recommendations explored for solving or avoiding these two forms of barriers in the context of research and vaccine administration include: 1) understanding more in depth the potential information derived from progress in neuroscience; 2) taking into account the role of religion and non-Western cultures in relation to a person-centred way of conceptualizing informed consent; 3 ) improving the readability and design of consent forms; 4) identifying the cultural and other bias of both the patient and the doctor/researcher; 5) evaluating the role of privacy in the collection of sensitive data connected to informed consent; 6) incorporating educationspecific strategies to improve patients' or participants' understanding of 
consent information; 7) initiating discussion of meningitis, HPV or RSV immunization and clearly explaining the benefits of infection prevention through immunization; 8) inviting questions at every step of the consent process; 9) acknowledging and addressing discrimination based on age and gender; 10) obtaining consent from legal representatives (in the case of children or pregnant women limited by mental defects or disorders); 11) protecting the privacy of participants enrolled in vaccine-related research; 12) acknowledging patients' or participants' own experiences with meningitis, RSV and/or HPV infection; 13) implementing procedures to assess patients' or participants' capacity to consent; 14) supporting parenting strategies and lifestyle practices that reduce and reverse predisposing risk factors to meningitis, RSV and HPV infection; 15) adopting individualized approaches to promote health protective behaviours (tailoring the consent process to reduce concerns relating to vaccine cost, pain, safety, side effects, perceived appropriateness to lifestyle and/or need for multiple doses) and 16) implementing a dynamic informed consent model with participant control, accompanied by appropriate privacy safeguards.

\section{A multicultural and interreligious perspective on informed consent}

The UNESCO International Bioethics Committee stressed in more than one occasion that an individual has to be informed as much as possible on the outcomes of the procedure in which she is involved in:

The close connection between autonomy and responsibility supposes that consent be freely given by the person concerned, the clearest possible information be provided, his/her faculties of comprehension be intact, that he/she has been able to assess the consequences of participating in a research project and the development of the entire process, as well as fully understanding the advantages and disadvantages of possible alternatives, also in terms of treatment. ${ }^{2}$

Aside from this analysis, various cultural and social variables are to be considered when assessing the ethical validity of the informed consent process. Oftentimes, such considerations might impinge upon the monolithic, individual-centred version of autonomy that we tend to give for granted in the Western contexts, creating a space for new versions of vulnerability-in which the vulnerable population is represented by those individuals unable to see their attitude and perception of autonomy as sufficiently represented by current legislations. In some scenarios, for example, we could use "communal autonomy" or "relational autonomy," a version of autonomy that sees the 
deliberation and the legitimacy of a decision to belong not only to a single person but rather the community to which one belongs (i.e. family). Often leaders of the community - nearly always family members - are those who make the decisions and their judgement is not questioned due to their age, expected wisdom and knowledge of the community's internal dynamics in place.

\section{Individual and relational autonomy}

In line with what just described, the words of Joseph Tham and Marie Letendre are particularly relevant to understand more accurately how some of our standard ways of conceptualizing the discussion around informed consent might not be as given as expected.

Cultural norms specify behavior. 'Honesty is an ideal value for most Americans, but it varies in strength as a real value for other cultures.' Honor is highly prized in the Japanese culture as is female purity in the Islamic world. Direct eye contact is avoided in several cultures, notably Asian and the Middle Eastern culture; the Navaho use silence to formulate their thoughts in order to give the most complete answer. Trust is given only to family members in the Gypsy culture. Masculine and feminine pronouns do not exist in Asian languages, and 'yes' does not always mean the affirmative since many cultures use the 'yes' as a way of avoiding an embarrassing 'no'. This is just a short list of cultural variables that inform and form communication styles. A cross-cultural health care ethic combines the tenets of patient-family centered care with an understanding of the social and cultural influences that affect the quality of medical services and treatment. Developing sensitivity to different cultures can make health care programs and activities attractive and interesting for a broader population base. In contrast, a lack of cultural sensitivity can deter people from using health care services. ${ }^{4}$

Hence, not all documents that assume that focusing on the individual might be sufficiently sensitive towards how one person with a cultural, religious or identitarian background might want (or is capable) to express her views, values and desires if disconnected from her community. In accepting this reality, it is equally important to bear in mind, as Loredana Persampieri rightly stresses, that - though contemplated - relational autonomy has no effective role in the shaping of informed consent in official forms. ${ }^{5}$

Seeking consent from an individual is necessary, even if the community is consulted, but the actual value of the consent of such individual, 
once the community has given its approval or disapproval, often raises concern. Nevertheless, such reasons should not lead to the conclusion that cultural considerations pave the way to situations where, exceptionally, for members of some groups communal autonomy may override individual autonomy. Conversely, we should always bear in mind that "respect for cultural diversity and pluralism should not be used to infringe fundamental freedoms nor any of the principles set out in the Declaration." "In this perspective, the Italian National Bioethics Committee suggests an interpretation of the concept of autonomy in terms of "relational autonomy," which may be better tailored to an intercultural approach aimed at accommodating the value of the community dimension in certain cultural settings (i.e. African tribes) and respect for the person. ${ }^{7}$

As the notion of informed consent relies on a set value of individual autonomy that not all cultures and approaches to life share, a patient's cultural disposition and past experiences with medical healthcare professionals will have an impact on the amount of trust that they can have in a vaccines' efficacy, for example. Although local culture may shape people's perception over time, people are more likely to trust experts that share a similar background, tradition, religion and culture with them. ${ }^{8}$ When working with ethnic minority patients, it is important to note that comprehension may also transcend simply linguistic barriers. The conceptualization of illness and cultural bias both plays a role in the ways that information is presented and understood. Thus, it is important to understand the role that culture plays in obtaining informed consent. ${ }^{9}$ In particular, in multicultural societies, where a large portion of the society is made up of immigrants with varying cultural backgrounds, there may be differing attitudes regarding the role of physicians. Moreover, the quality of informed consent may be dependent on the relationship between a physician and their patient.

To improve the physician-patient relationship and for the consent gained to be effective, there has to be a partnership based on openness, trust and good communication between the two parties. ${ }^{10}$ Individual's religious beliefs or related cultural values can lead to questions and concerns that health professionals, unfamiliar with the religion or culture, have not encountered before. It has been shown that culture (which can also include religious and spiritual backgrounds) can impact one's vulnerability to infectious diseases. Rejecting treatments or prevention measures due to religious or cultural values is not a new phenomenon; there have been reports of vaccines-preventable outbreaks in religious schools, congregations and religious communities. ${ }^{11}$ As a case study, the World Health Organization reported that in a region in Nigeria $16 \%$ of the children were vaccinated 
against polio. The reason for the low vaccination rates is that the community is predominantly Muslim, and they believe that the polio drops are used as a tool to sterilize the children. Likewise, a study from the Netherlands has shown that municipalities with high orthodox protestant domination have lower vaccination rates compared to municipalities without an orthodox protestant domination. ${ }^{12}$

Regarding the way informed consent notion interacts with biomedical research, some of the key questions that we want to address here are:

a) How much of the notion of informed consent is applied in one's tradition? And in which way?

b) Can or should we have different informed consent forms for differently vulnerable populations?

c) Do all traditions agree with the general principles behind informed consent (i.e. the prioritization of individual autonomy)? If not, what alternative values/approach could support widespread vaccination, for example?

\section{Recommendations}

As the main objective of this project is to identify the ethical gaps, barriers and challenges currently present in obtaining informed consent from patients in different, challenging contexts and address the issues with some practical suggestions for future policies, two main deliverables can be extracted from the inputs here analysed. They should be further expanded and taken into consideration when developing new models and forms that aim at providing convincing guidelines for the informed consent process.

The first aspect to take into account is the role of religious keywords. Implementation of some key terms directly refers to some religious traditions. For example, kosher or halal in vaccines, or reference to xiaodao and dadao as notions helpful to conceptualize better why we, as single individuals, should behave in a certain way in relation to society. Not only ensuring the "religious approval" from different traditions will increase the trust towards doctors and researchers, but it will also make more evident and immediate in the eyes of the believer terms that will help him filling up required forms and documents with more conviction, speeding up the process of sharing scientific information.

The second point is that international accepted notions and values, such as human duties, ${ }^{13}$ should be considered when discussing informed consent, not only human rights. Where possible, use the specific tradition to reinforce the duties towards society as a whole. For example, the principle 
of the public interest (maslahat al-ummah) that sees vaccines as a way to protect others in Islam. Or the idea of dharma in the Hindu tradition in relation to laws and duties towards society (stressed by many other traditions through different concepts, notions and approaches but still very similar in practice).

These are the final recommendations to increase the effectiveness of multicultural and interreligious perspective:

- Taking into account that not all traditions and religions give the same level of importance to the individual-centred version of autonomy at the base of the informed consent form conceived to be signed by a single individual.

- Implementing some key terms directly referring to some cultural and religious traditions. Considering other key notions such as human duties, not only human rights.

- Fostering participation of trained cross-cultural professionals as members of the ethical research committees to validate cultural and religious concerns during research. Increasing the diversity of the healthcare professionals, improve the opportunity to have individuals capable to filter more directly certain scientific notions into some religious and traditional guidelines.

- Stimulating the composition of cross-cultural research teams, facilitating an understanding of cultural and religious diversity when recruiting and when carrying out research in patients with different cultural backgrounds and religious convictions.

- Capturing the patient religious or cultural background to allow the researcher to introduce appropriate religious and cultural concepts (or terms), when necessary, in the IC form and during the communication process, facilitating the understanding, trust and acceptance of believers towards social value of science and research, improving the acceptance rate of participation in clinical trial.

- Changing the categorization of the patients focusing on a shared common cultural identity. Healthcare professionals should ask questions about other social identities to shift their attention from the patient's ethnicity or religious background helping to reduce racial or cultural biases to improve recruitment of minorities.

- Fostering the religious and community leaders' analysis and possible support or approval of specific scientific biomedical research (i.e. specific therapy or vaccines) so that their support might illuminate believers and increase the trust towards doctors and researchers as well as participation in clinical trials. 


\section{Notes}

1 The full report can be accessed here: https://i-consentproject.eu/wp-content/uploads/ 2019/01/D1.4-Ethical-issues-concerning-informed-consent-in-translationalclinicalresearch-and-vaccination.pdf.

2 UNESCO IBC (2008), 15, https://unesdoc.unesco.org/ark:/48223/pf0000178124.

3 Rachel E. Spector, Cultural Diversity in Health and Illness (Upper Saddle River, NJ: Prentice Hall, 2000). Antonella Surbone, "Telling the Truth to Patients with Cancer: What Is the Truth?," Oncology 7 (2006): 994-950.

4 Joseph Tham and Marie-Catherine Letendre, "Health Care Decision Making: Cross-Cultural Analysis of the Shift from the Autonomous to the Relational Self," The New Bioethics 20, no. 2 (2014): 180-181.

5 Loredana Persampieri, i-CONSENT December Workshop, Rome, Italy (2017). (unpublished).

6 UNESCO Declaration on Bioethics and Human Rights (2005), Art. 12.

7 Italian Committee for Bioethics (2017), 38, http://bioetica.governo.it/media/ 1391/p128_2017_immigrazione-e-salute it.pdf.

8 Dan M. Kahan, et al., "Who Fears the HPV Vaccine, Who Doesn't, and Why? An Experimental Study of the Mechanisms of Cultural Cognition," Law and Human Behavior 34, no. 6 (2010): 501-516.

9 Simon Dein and Kamaldeep Bhui, "Issues Concerning Informed Consent for Medical Research among Non-Westernized Ethnic Minority Patients in the UK," Journal of the Royal Society of Medicine 98, no. 8 (2005): 354-356.

10 General Medical Council, Consent: Patients and Doctors Making Decisions Together (2008), www.gmc-uk.org/-/media/documents/gmc-guidance-for-doctors--consent---english_pdf-48903482.pdf.

11 Tami Lynn Thomas, et al., "Parental Human Papillomavirus Vaccine Survey (PHPVS): Nurse-Led Instrument Development and Psychometric Testing for Use in Research and Primary Care Screening," Journal of Nursing Measurement 21, no. 1 (2013): 96-109.

12 John D. Grabenstein, "What the World's Religions Teach, Applied to Vaccines and Immune Globulins," Vaccine 31, no. 16 (2013): 2011-2023.

13 Fundación Valecia Tercer Milenio, Declaration of Human Duties and Responsibilities (1999), http://unesdoc.unesco.org/Ulis/cgi-bin/ulis.pl?catno=188520\&g $\mathrm{p}=\& \operatorname{lin}=1 \& 11=\mathrm{f}$. 